\title{
Etiological Factors and Clinical Profile of Acute Kidney Injury
}

\section{in Medical Intensive Care Unit}

\author{
Dr. Mekha K. Mathew ${ }^{\star, \dagger, 1}$, Dr. T. R. Radha ${ }^{2}$ \\ ${ }^{1}$ Senior Resident, Department Of General Medicine, Government Medical College, Kottayam, Kerala, India ${ }^{2}$ Additional \\ Professor, Department Of General Medicine, Government Medical College, Kottayam, Kerala, India \\ DDOI: https:// doi.org/10.15520/jcmro.v2i11.235
}

Accepted 26-11-2019; Received 10-10-2019; Publish Online 27-11-2019

\author{
Reviewed By: \\ Dr. K. Daniel \\ Department: \\ Reviewer/CMRO
}

\begin{abstract}
Background and Objectives: Data on the etiology, management practices and outcome in patients admitted to intensive care units in Kerala has not been studied in depth. The present study was undertaken to identify the etiologic factors and outcome of patients with Acute Kidney Injury admitted in the Medical Intensive Care Unit.

Methods: Cross sectional study, done in 150 consecutive patients admitted to MICU, Government Medical College, Kottayam meeting the AKIN creatinine criteria for AKI over a period of 12 months.

Results and Discussion: The most common diagnosis in the patients admitted with AKI in MICU was Leptospirosis (22\%) followed by undifferentiated fever (21\%) and sepsis seen in $17 \% .45 \%$ of the patients required dialysis, among which $24 \%$ underwent CRRT while the $76 \%$ underwent hemodialysis. Out of 150 cases $59 \%$ survived and $41 \%$ expired. Mortality was more in the patients in this study with undifferentiated acute febrile illness (43.8\%) and leptospirosis (36.4\%).

Conclusion: The most common etiology for AKI was leptospirosis (22\%) followed by undifferentiated fever (21\%). Mortality was $41 \%$ in the patients in the study group. Requirement of ventilator support and inotropic supports were independent predictors of mortality.
\end{abstract}

Key words: Leptospira; $A K I ; M I C U$

\section{INTRODUCTION:}

Acute kidney injury (AKI) complicates $5-7 \%$ of acute care hospital admissions and up to $30 \%$ of admissions to the intensive care unit. [1] The tropical zone which is geographically limited by the Tropics of Cancer and Capricorn, is an area with extreme heterogeneity in terms of ethnic composition, as well as the socioeconomic and developmental status. Acute kidney injury remains one of the most enigmatic problems in these regions with high incidence rates $(25 \%-80 \%)$ especially in the intensive care units.

There are very few studies on the etiologic factors of acute kidney injury in the intensive care units in South India. Unlike in the developed nations, were sepsis is a predominant etiologic factor; in high rainfall receiving regions of the tropics - like Kerala, infections like leptospirosis form a major cause of AKI.

\footnotetext{
* Corresponding author.

$\dagger$ Email: drmekhamathew333@gmail.com
}

\section{OBJECTIVE:}

To study the etiologic factors, clinical profile and outcome of patients with Acute Kidney Injury admitted in Medical intensive care unit.

\section{MATERIAL AND METHODS:}

Study Design : Cross sectional analytical study Study Setting : Medical Intensive Care Unit, Government Medical College, Kottayam

Duration of Study : 12 Months

Study Population : Patients with AKI diagnosed by AKIN Creatinine Criteria

\section{Sample Size}

$\mathrm{n}=(\mathrm{Z} \alpha)^{2} \mathrm{pq} / \mathrm{d}^{2}$

$\mathrm{Z} \alpha=1.96$ for $\alpha$ at $5 \%$ level of significance

$\mathrm{p}=$ prevalence of sepsis causing AKI- 38.6\%

(Based on Eswarappa et al on spectrum of AKI in critically ill patients [2]

$$
q=100-p
$$


$\mathrm{d}=$ absolute precision $[3]$

$(4 \times 38.6 \times 61.4) / 8 \times 8=148$

Minimum required sample size - 150

Study Tool : Performa based on case records

Inclusion Criteria : atients admitted to MICU within the study period with diagnosis of Acute Kidney Injury by AKIN creatinine criteria

Exclusion Criteria : Patients with Chronic Kidney disease

Study Procedure : 150 consecutive patients admitted to Medical Intensive Care Unit during my study period meeting the AKIN creatinine criteria for acute kidney injury was included in this study. All patients or their relatives were interviewed as per the prepared performa and then complete clinical examination was done.

The following investigations were performed: blood routine, random blood sugar, renal function tests, liver function tests, serum electrolytes, serum uric acid, INR, blood pH, urine analysis, ECG, chest $\mathrm{X}$ ray, ultrasonography of abdomen for all cases and specific tests for diagnosis in special cases. The data collected was entered in Microsoft excel and analyzed using SPSS software. Associations between various factors were assessed using chi square test for qualitative variables and t test/ANOVA for quantitative variables. Appropriate non parametric tests were applied wherever required. The level of statistical significance was taken as $\mathrm{p}$ value less than 0.05 .

Ethical consideration: The above mentioned study was conducted in this institution after obtaining due ethical clearance from the Ethical Committee of Government Medical College, Kottayam. Informed consent was obtained from cases in the study.

\section{THEORY:}

AKI affects patients worldwide, leading to decreased survival, and sometimes to new onset of CKD. Outcomes in AKI are influenced by the underlying disease, the severity and duration of renal impairment and by the baseline characteristics of the patient. The cause of AKI is often multi-factorial.

The role of sepsis in AKI has been well documented in western literature, causing nearly $50 \%$ of the AKI cases in few studies. [4-6] Sepsis is associated with high burden of illness, greater abnormality in acute physiology \& laboratory findings \& greater non-renal organ failure [1-3] . Approximately $25-35 \%$ of patients with severe sepsis and $40-55 \%$ of patients with septic shock die within 30 days $[1,2]$.

Other causes include acute gastroenteritis, nephrotoxic drugs, cardiac causes and hepatic causes. Hypertension, diabetes and coronary artery disease are predominant comorbid conditions. Fidel Barrantes et al concluded that the Acute Kidney Injury Network (AKIN) definition of acute kidney injury predicts hospital mortality, need for renal replacement therapy and prolonged hospital stay in critically ill patients. [4]

The true picture of AKI in the tropics is poorly understood due to the late presentation of patients to tertiary centres. Tropical infectious diseases are an important cause of admission to ICU in Kerala, and diseases like leptospirosis have a high propensity to cause AKI.

\section{CASE DEFINITIONS}

Acute Kidney Injury:

Defined by AKIN Creatinine Criteria

Stage 1: Increase of S Cr $\geq 0.3 \mathrm{mg} / \mathrm{dl} /$ increase of S Cr $\geq 150 \%$ to $200 \%$ over $48 \mathrm{hrs}$

Oliguria urine output $<0.5 \mathrm{ml} / \mathrm{kg} / \mathrm{hr}$ over $6 \mathrm{hrs}$

Stage 2: Increase of S Cr $\geq 200 \%$ to $300 \%$ over 48 hours Oliguria urine output $<0.5 \mathrm{ml} / \mathrm{kg} / \mathrm{hr}$ over $12 \mathrm{hrs}$

Stage 3: Increase of S Cr $\geq 300 \%$

$\mathrm{S} \mathrm{Cr} \geq 4 \mathrm{mg} / \mathrm{dl}$ and acute rise $\geq 0.5 \mathrm{mg} / \mathrm{dl}$

Initiation of RRT [7]

A diagnosis of sepsis/septic shock was made where the primary reason for admission was a sepsis related illness, and included sepsis associated with pneumonia, gastrointestinal disease, urinary tract infections, central nervous system infections, soft tissue infections and sepsis of undetermined source based on a qSOFA score $>2$ in presence of documented infection. A cardiac diagnosis was made where the primary reason for admission was cardiogenic shock, cardiac arrest, congestive cardiac and acute myocardial infarction.

A respiratory diagnosis encompassed aspiration syndrome, exacerbations of chronic obstructive pulmonary disease or asthma. A diagnosis of gastrointestinal diagnosis included chronic liver disease, pancreatitis and acute diarrheal disease. Metabolic/poisoning diagnoses included causes of metabolic coma, diabetic ketoacidosis, drug overdose or other endocrinopathies. Primary neurologic diagnoses included stroke or other neurological causes for coma. A diagnosis of Dengue by IgM Dengue ELISA test. A diagnosis of probable Leptospirosis based on System of scoring using the Modified Faine's Criteria [8]

\section{RESULTS:}

\section{Gender distribution}

$73.3 \%$ of the patients in the study group were males and $26.7 \%$ were females.

\section{Age distributionTable 1}

Mean age was 49.6 years with a standard deviation of 16.5. Youngest and the oldest of the study population had 15 and 88 years of age respectively.

\section{Presenting symptomsFigure 1}

Fever was the most common presenting symptom seen in $108(72 \%)$ patients, decreased urine output in $72(48 \%)$ patients, breathlessness in $68(45 \%)$ patients, and myalgia in $64(43 \%)$ patients.

Other common presenting symptoms were headache, cough, altered sensorium and abdominal pain seen in 35 (23\%), 30 (20\%), 28 (19\%) and 28 (19\%) respectively.

4. Nephrotoxic drug intakeTable 2

Only 10 patients that accounts for $7 \%$ had h/o nephrotoxic drug usage. NSAID $(2.7 \%)$ was the most common one implicated used by 4 patients prior to development of AKI. 
Table 1. Age distribution

\begin{tabular}{|c|c|c|c|}
\hline $\begin{array}{l}\text { Age group } \\
\text { (years.) }\end{array}$ & $\begin{array}{l}\text { SEX } \\
\text { Male }\end{array}$ & Female & Total \\
\hline$<24$ & $11(10.0 \%)$ & $2(5.0 \%)$ & $13(8.7 \%)$ \\
\hline $25-34$ & $14(12.7 \%)$ & $5(12.5 \%)$ & $19(12.7 \%)$ \\
\hline $35-44$ & $20(18.2 \%)$ & $4(10.0 \%)$ & $24(16.0 \%)$ \\
\hline $45-54$ & $20(18.2 \%)$ & $10(25.0 \%)$ & $30(20.0 \%)$ \\
\hline $55-64$ & $22(20.0 \%)$ & $9(22.5 \%)$ & $31(20.7 \%)$ \\
\hline $65-74$ & $15(13.6 \%)$ & $8(20.0 \%)$ & $23(15.3 \%)$ \\
\hline 75 \&above & $8(7.3 \%)$ & $2(5.0 \%)$ & $10(6.7 \%)$ \\
\hline Total & $110(100.0 \%)$ & $40(100.0 \%)$ & $150(100.0 \%)$ \\
\hline
\end{tabular}

\section{PRESENTING SYMPTOMS}

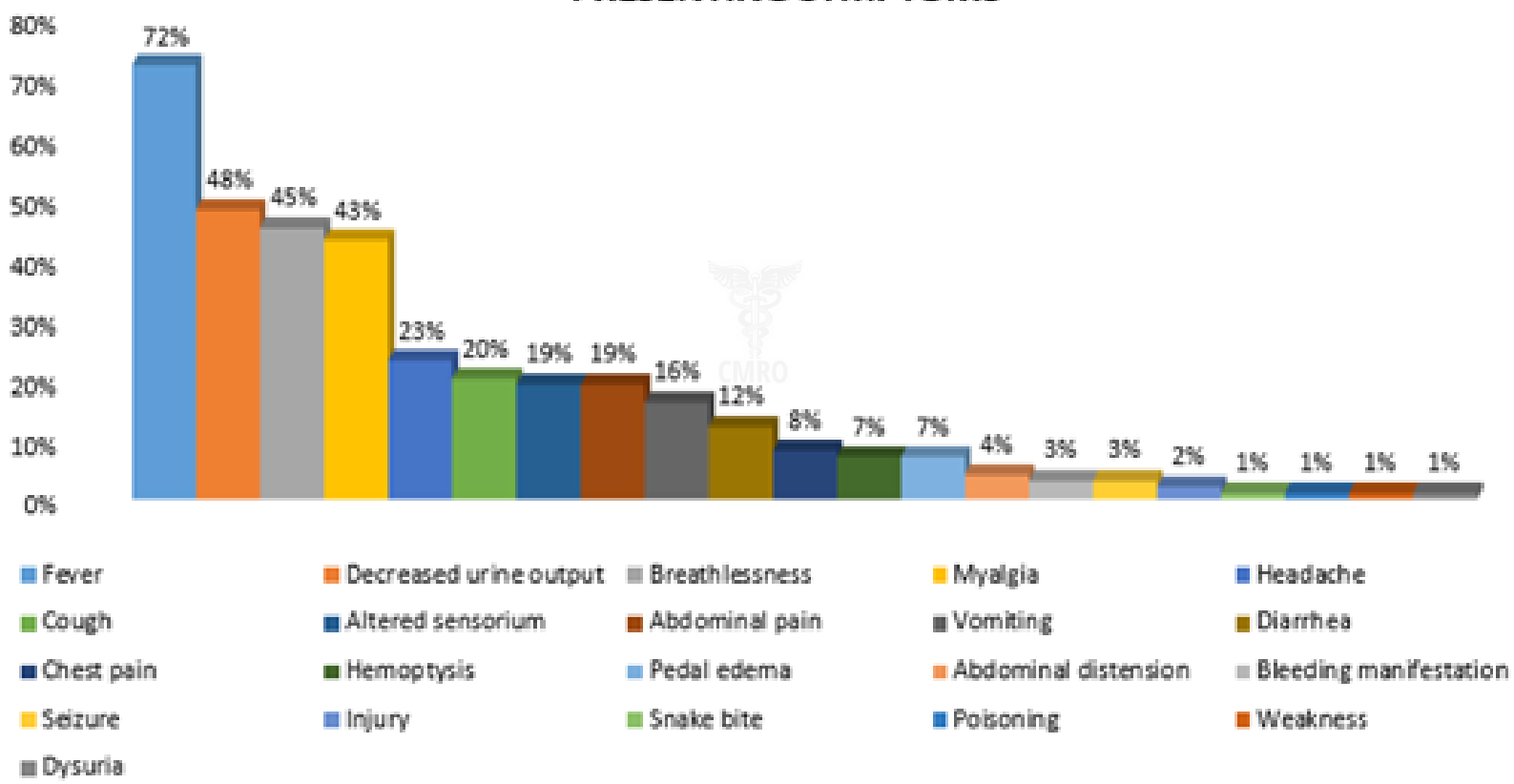

Figure 1. Presenting symptoms

Table 2. Nephrotoxic drug intake

\begin{tabular}{lll}
\hline Drugs & Frequency & Percentage \\
No & 140 & $93.3 \%$ \\
NSAID & 4 & $2.7 \%$ \\
Lithium & 2 & $1.3 \%$ \\
ARB & 2 & $1.3 \%$ \\
Herbal & 1 & $0.7 \%$ \\
Cocaine & 1 & $0.7 \%$ \\
Total & 150 & $100 \%$ \\
\hline
\end{tabular}


Table 3. Etiologic factors

\begin{tabular}{lll}
\hline Etiologic factors & Frequency & Percentage \\
Leptospirosis & 33 & $22 \%$ \\
Undifferentiated acute febrile illness & 32 & $21.3 \%$ \\
Sepsis & 26 & $17.3 \%$ \\
Respiratory diagnosis & 17 & $11.3 \%$ \\
Cardiac diagnosis & 14 & $9.3 \%$ \\
Gastrointestinal & 13 & $8.7 \%$ \\
Metabolic/poisoning diagnoses & 7 & $4.7 \%$ \\
Primary neurologic diagnoses & 3 & $2 \%$ \\
Dengue & 3 & $2 \%$ \\
Drug & 1 & $0.7 \%$ \\
Snake bite & 1 & $0.7 \%$ \\
Total & 150 & $100.0 \%$ \\
\hline
\end{tabular}

\section{ETIOLOGIC FACTORS}

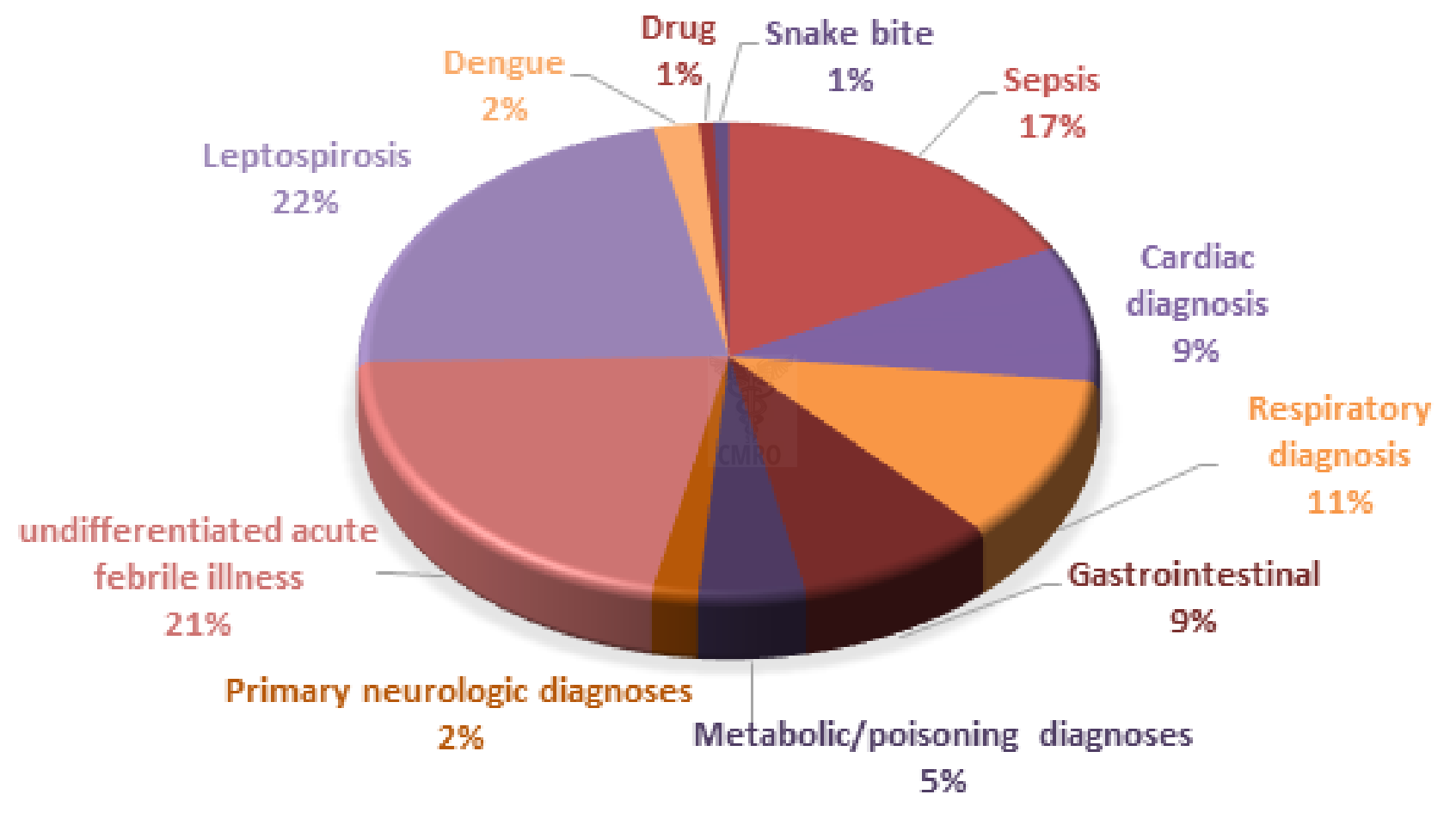

Figure 2. Etiologic factors

\section{Etiologic factorsTable 3Figure 2}

The most common etiology of AKI in the patients in this study group was leptospirosis seen in 33 patients which accounts to $22 \%$ followed by undifferentiated fever seen in 32 patients $(21 \%)$ and sepsis seen in 26 patients (17\%).

6. Stage of acute kidney injury by the AKIN creatinine criteriaFigure 3

The most common stage of AKI in the patients in this study group on admission to the medical intensive care unit was stage 3 seen in 97 patients which accounts to $64.7 \%$.

\section{Dialysis RequirementFigure 4}

68 out of the 150 patients in the study group required dialysis, which accounts to $\mathbf{4 5 \%}$. Among the patients in the study group who underwent dialysis 52 patients $(76.5 \%)$ underwent intermittent hemodialysis while 16 patients $(23.5 \%)$ underwent CRRT.

\section{Indication For DialysisTable 4}

The predominant indication for dialysis in the patients in the study group was metabolic acidosis and anuria in $52.9 \%$ 


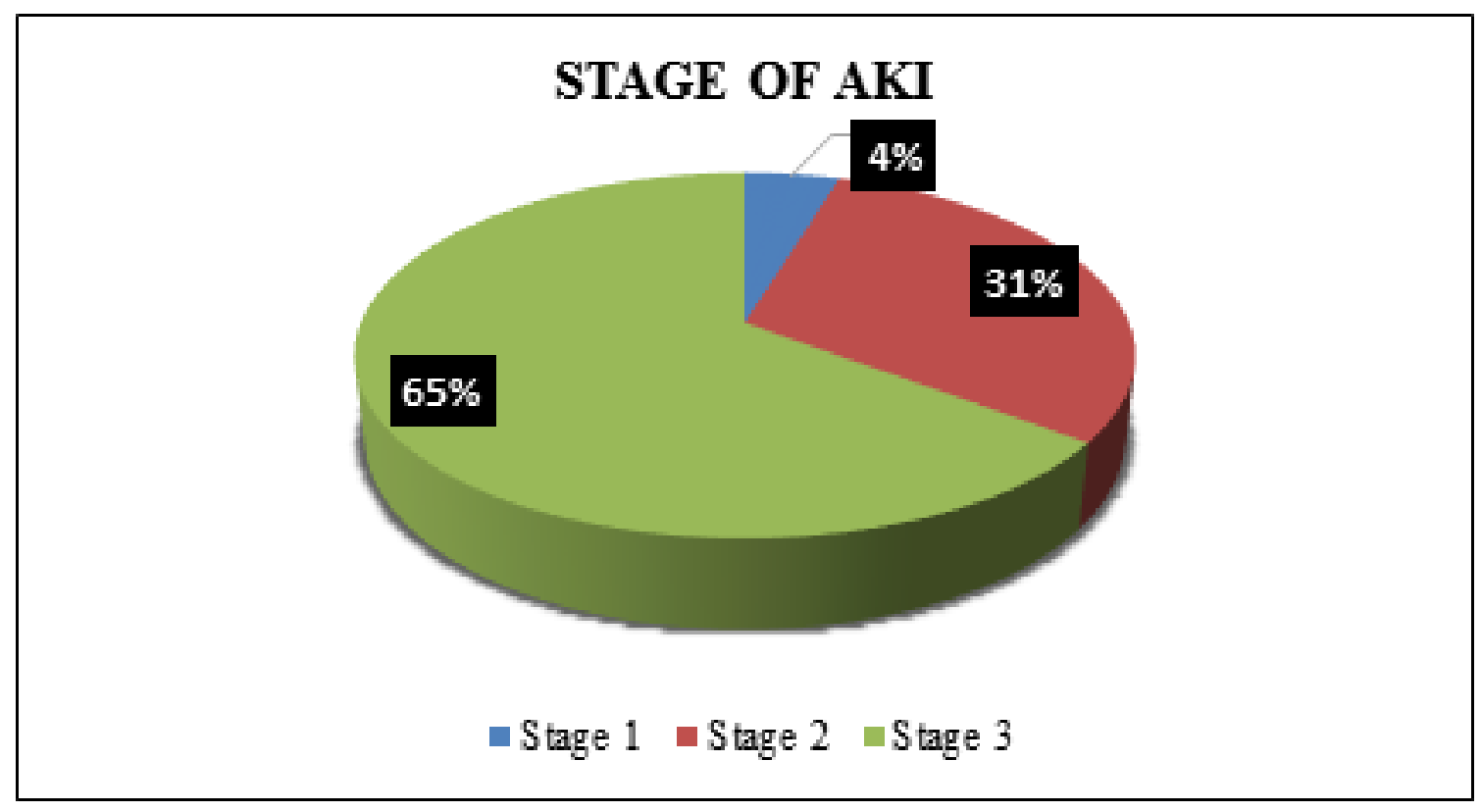

Figure 3. Stage of acute kidney injury

\section{DIALYSIS TYPE}

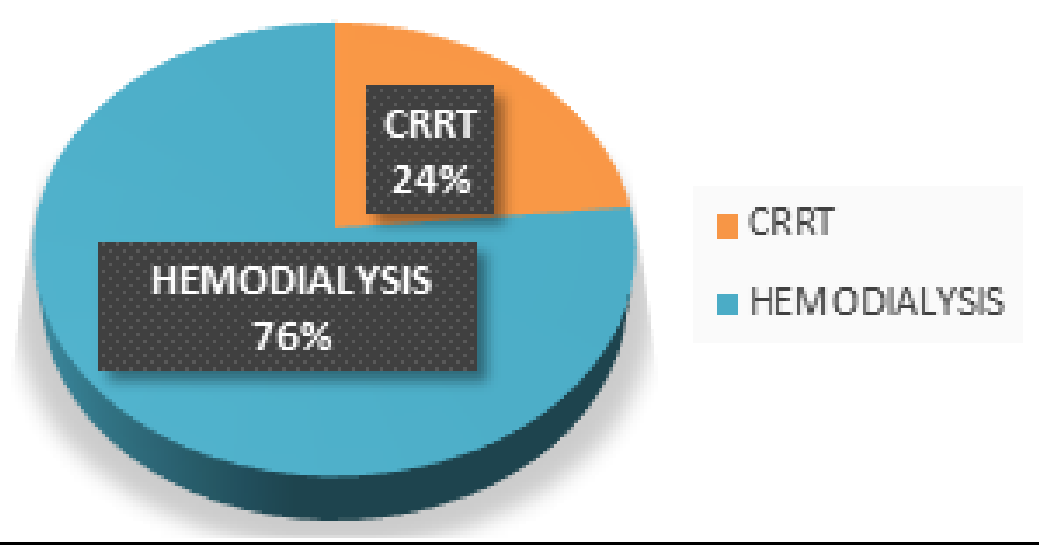

Figure 4. Dialysis type

Table 4. Indication for dialysis

\begin{tabular}{lll}
\hline & Frequency & Percentage \\
Metabolic acidosis alone & 9 & $13.2 \%$ \\
Hyperkalemia & 1 & $1.5 \%$ \\
Anuria alone & 22 & $32.4 \%$ \\
Metabolic acidosis and anuria & 36 & $52.9 \%$ \\
Total & 68 & $100.00 \%$ \\
\hline
\end{tabular}


of the patients.

\section{Ventilation RequirementFigure 5}

66 out of the 150 patients which accounts to $56 \%$ required invasive ventilation.

\section{Inotrope RequirementTable 5}

125 of the 150 patients in the study group which accounts for $83 \%$ required inotropic support.

\section{Mortality Figure 6}

Mortality was $41 \%$ in the patients in the study group, 62 patients out of 150 expired.

12. Association Between Aki Stage And MortalityTable 6

$53 \%$ of those with stage 3 AKI expired compared to no mortality in the stage 1or2.

13. Association Between Inotrope Use And MortalityTable 7

Mortality was more in the patients in this study requiring inotrope support. $48 \%$ of those with inotropic support expired.

14. Association Between Ventilation And MortalityTable 8

Mortality was more in the patients in this study requiring ventilator support.

15. Association Between Diagnosis And OutcomeTable 9Figure 7

Mortality was more in the patients in this study with undifferentiated acute febrile illness, $43.8 \%$ and leptospirosis, $36.4 \%$.

16. Association Between Diagnosis And Stage Of AKITable 10Figure 8

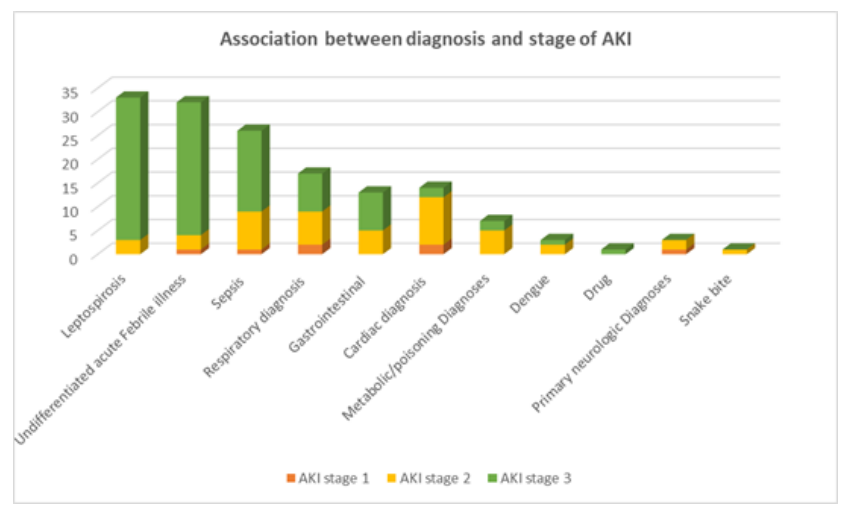

Figure 8. Association between diagnosis and stage of AKI

$90.9 \%$ of those with leptospirosis and $87.5 \%$ of those with undifferentiated febrile illness presented in AKI stage 3.

17. Logistic Regression Analysis Of Outcome PredictorsTable 11

By logistic regression analysis of the factors having association with mortality, requirement of ventilator support and inotropic supports were independent predictors of mortality.
6 DISCUSSION:

\section{AGE AND GENDER}

A trend towards an increasing number of AKI cases among male patients was observed compared to female patients [ $73 \%$ and $27 \%$ respectively] in this study.

Compared with all ICU admissions, patients with AKI were found in male gender in Basu et al, Cruz et al and Daher et al [9-11] . The reasons for such gender differences are unclear but are likely associated with work activities.

Majority of patients in this study belonged to 55-65 years (21\%) followed by $45-55$ years (20\%). Mean age was 49.6 years with standard deviation 16.5. The population of patients with AKI in developing tropical countries is younger (30-40 years of age) than that reported in developed temperate countries (60-70 years of age) like Angus et al [12]

\section{CLINICAL FEATURES}

Fever was the most common presenting symptom (72\%) followed by decreased urine output (48\%), breathlessness (45\%), and myalgia (43\%). Predominant presenting features included fever (89.6\%), oliguria (70.4\%), and breathlessness (40.4\%) as in Mehta et al [13].

Only $7 \%$ of the patients in this study had h/o nephrotoxic drug usage. NSAID (2\%) being the most common one implicated.

The most common general examination finding in the patients in this study was icterus $(52 \%)$ followed by conjunctival congestion $(22 \%)$. The most common systemic examination finding in patients in this study was basal crepitations (40\%). In Eswarappa et al, edema was seen in 28.0\%, pallor in $20.0 \%$ and icterus in $21.0 \%$ of the patients [6]

The mean serum creatinine in the patients in this study on admission to the Medical ICU was $3.72 \mathrm{mg} / \mathrm{dL}$ with a standard deviation of 1.65 . Mean serum creatinine was 3.64 with $S D$ of 3.1 in a study by Maulita et and $10.18 \pm 5.19$ $\mathrm{mg} / \mathrm{dl}$ in a study by Ahmed et al [14]

\section{DIAGNOSIS}

The most common diagnosis in the patients in this study was leptospirosis $(22 \%)$ followed by undifferentiated fever $(21 \%)$ and sepsis seen in $17 \%$.

In contrast to sepsis, drugs and cardiogenic shock in the developed world, acute tubular necrosis (ATN) due to community-acquired infections remains the commonest cause of AKI in the tropics. Chug, Medve and Angus et al [15-17]

The main causes of ICU hospitalization were acquired immunodeficiency syndrome (AIDS)-related diseases (28.6\%), pneumonia 13\%), leptospirosis (11.6\%) in Daher et al [16] It was scrub typhus (51.2\%), falciparum malaria (10.4\%), and enteric fever (8.7\%) in study by Basu et al [14]. Unlike in our study the predominant cause of AKI in most studies in tropical areas was due to malaria.

$90.9 \%$ of those with leptospirosis and $87.5 \%$ of those with undifferentiated febrile illness presented in AKI stage 3. Significant association was identified between the etiology of AKI and the stage of presentation on admission to the medical intensive care unit.

Significant association was identified between etiology of $\mathrm{AKI}$ and the need for dialysis. Dialysis was required in 


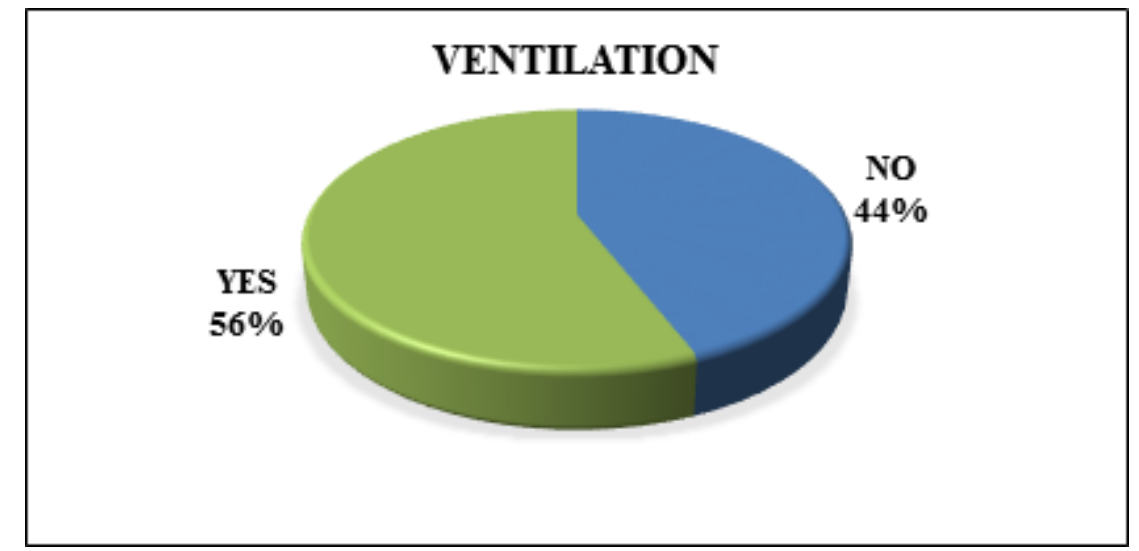

Figure 5. Ventilation requirement

Table 5. Inotrope requirement

\begin{tabular}{lcc}
\hline & Frequency & Percentage \\
No & 25 & $16.70 \%$ \\
Yes & 125 & $83.30 \%$ \\
Total & 150 & $100.00 \%$ \\
\hline
\end{tabular}

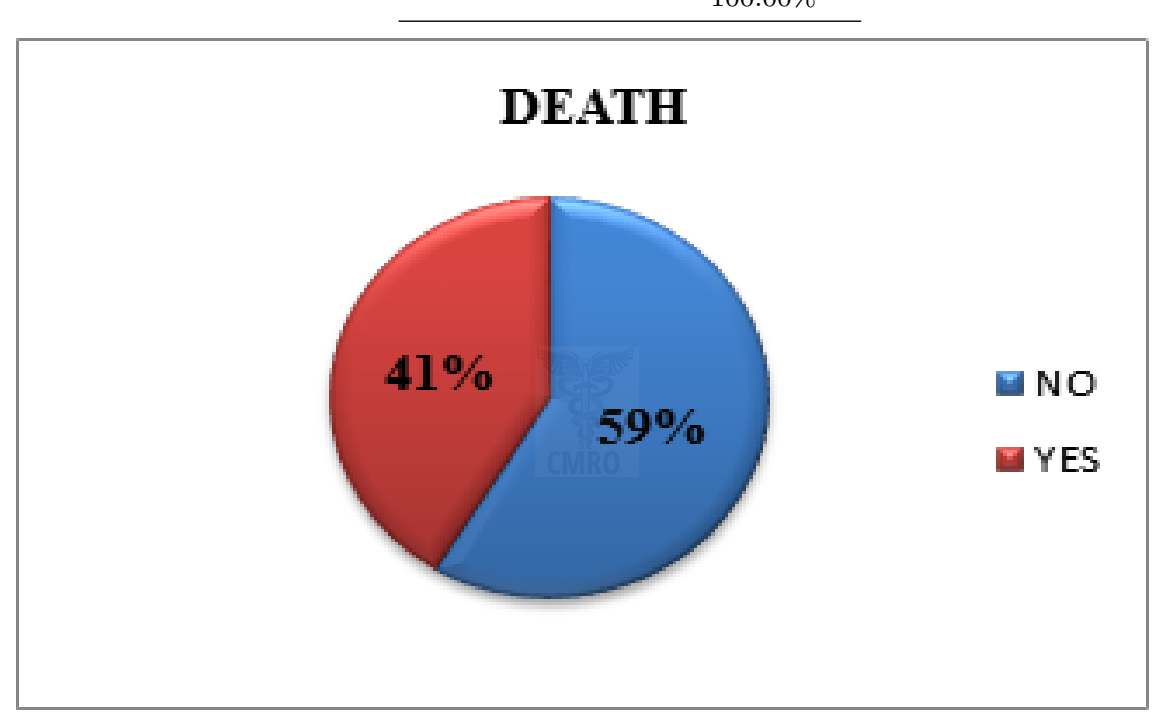

Figure 6. Mortality

Table 6. Association between AKI stage and mortality

\begin{tabular}{|c|c|c|c|}
\hline & & \multicolumn{2}{|c|}{ Outcome } \\
\hline & & Survived & Expired \\
\hline \multirow{6}{*}{ AKI stage } & \multirow{2}{*}{ Stage 1} & 5 & 0 \\
\hline & & $100.0 \%$ & 0 \\
\hline & \multirow{2}{*}{ Stage 2} & 28 & 0 \\
\hline & & $100.0 \%$ & 0 \\
\hline & \multirow{2}{*}{ Stage 3} & 55 & 62 \\
\hline & & $47.0 \%$ & $53.0 \%$ \\
\hline \multirow{2}{*}{\multicolumn{2}{|c|}{ Total }} & Number & 62 \\
\hline & & $\%$ & $41.3 \%$ \\
\hline
\end{tabular}


Table 7. Association between inotrope use and mortality

\begin{tabular}{|l|c|c|c|}
\hline \multirow{2}{*}{} & \multicolumn{2}{|c|}{ Outcome } & \multirow{2}{*}{} \\
\cline { 2 - 3 } & Survived & Expired & Total \\
\hline \multirow{3}{*}{ Inotropic support absent } & 23 & 2 & 25 \\
\cline { 2 - 4 } & $92.0 \%$ & $8.0 \%$ & $100.0 \%$ \\
\cline { 2 - 4 } Inotrope support present & 65 & 60 & 125 \\
\hline \multirow{2}{*}{ Total } & $52.0 \%$ & $48.0 \%$ & $100.0 \%$ \\
\cline { 2 - 4 } & $58.7 \%$ & 62 & 150 \\
\hline
\end{tabular}

Pearson chi-square Value - 13.746

$P$ value -0.000

Table 8. Association between ventilation and mortality

\begin{tabular}{|c|c|c|c|}
\hline \multirow{4}{*}{ Ventilator support absent } & \multicolumn{2}{|c|}{ Outcome } & \multirow{2}{*}{ Total } \\
\cline { 2 - 3 } & Survived & Expired & \multirow{2}{*}{66} \\
\cline { 2 - 3 } & 63 & 3 & \multirow{2}{*}{84} \\
\hline \multirow{2}{*}{ Ventilator support present } & $95.5 \%$ & $4.5 \%$ & 59 \\
\cline { 2 - 3 } Total & $29.8 \%$ & $70.2 \%$ & \multirow{2}{*}{150} \\
\cline { 2 - 3 } & $58.7 \%$ & $41.3 \%$ & \\
\hline
\end{tabular}

Pearson chi-square -65.777

$P$ value -0.000

Table 9. Association between diagnosis and Outcome

\begin{tabular}{|c|c|c|c|}
\hline & & & \\
\hline & & Survived & Expired \\
\hline & Sepsis & 16 & 10 \\
\hline & & $61.50 \%$ & $38.50 \%$ \\
\hline & Cardiac diagnosis & 7 & 7 \\
\hline & & $50.00 \%$ & $50.00 \%$ \\
\hline & Respiratory diagnosis & 11 & 6 \\
\hline & & $64.70 \%$ & $35.30 \%$ \\
\hline & Gastrointestinal & 9 & 4 \\
\hline & & $69.20 \%$ & $30.80 \%$ \\
\hline & Metabolic/poisoning Diagnoses & 3 & 4 \\
\hline & & $42.90 \%$ & $57.10 \%$ \\
\hline Diagnosis & Primary neurologic Diagnoses & 1 & 2 \\
\hline & & $33.30 \%$ & $66.70 \%$ \\
\hline & Undifferentiated acute Febrile illness & 18 & 14 \\
\hline & & $56.30 \%$ & $43.80 \%$ \\
\hline & Leptospirosis & 21 & 12 \\
\hline & & $63.60 \%$ & $36.40 \%$ \\
\hline & Dengue & 2 & 1 \\
\hline & & $66.70 \%$ & $33.30 \%$ \\
\hline & Drug & 0 & 1 \\
\hline & & $0.00 \%$ & $100.00 \%$ \\
\hline & Snake bite & 0 & 1 \\
\hline & & $0.00 \%$ & $100.00 \%$ \\
\hline & & 88 & 62 \\
\hline Total & & $58.70 \%$ & $41.30 \%$ \\
\hline
\end{tabular}

$81.3 \%$ among those with undifferentiated febrile illness and $78.8 \%$ among those with leptospirosis and the association was significant by Chi square test.

Leptospirosis with AKI admitted to ICU had a significantly higher frequency of severe AKI 73.2 \% and a higher prevalence of dialysis requirement (57.3\%) in a study by De Francesco et al [18]

\section{MANAGEMENT}

The most common stage of AKI in the patients in this study group on admission to the medical intensive care unit was stage 3 in $64.7 \%$. Unlike in a study by lopes et al in which AKI occurred in $50.4 \%$ of patients - 21.1\% with Stage 1, $10.1 \%$ with Stage 2 and 19.2\% with Stage 3 .
$45 \%$ of the patients in the study group required dialysis, among which $24 \%$ underwent CRRT while the $76 \%$ underwent hemodialysis.

In a study conducted by Bernieh b et al. 58\% of patients were managed conservatively while $42 \%$ patient were managed with dialysis which is comparable with present study [12]

The predominant indication for dialysis in the patients in this study group was metabolic acidosis with anuria. The most common indication for dialysis was refractory fluid overload (89.4\%), followed by uremic signs and symptoms $(61.1 \%)$ in study by Ibrahim et al [19] , probably because CKD patients were also included. 


\section{Association between diagnosis and death}

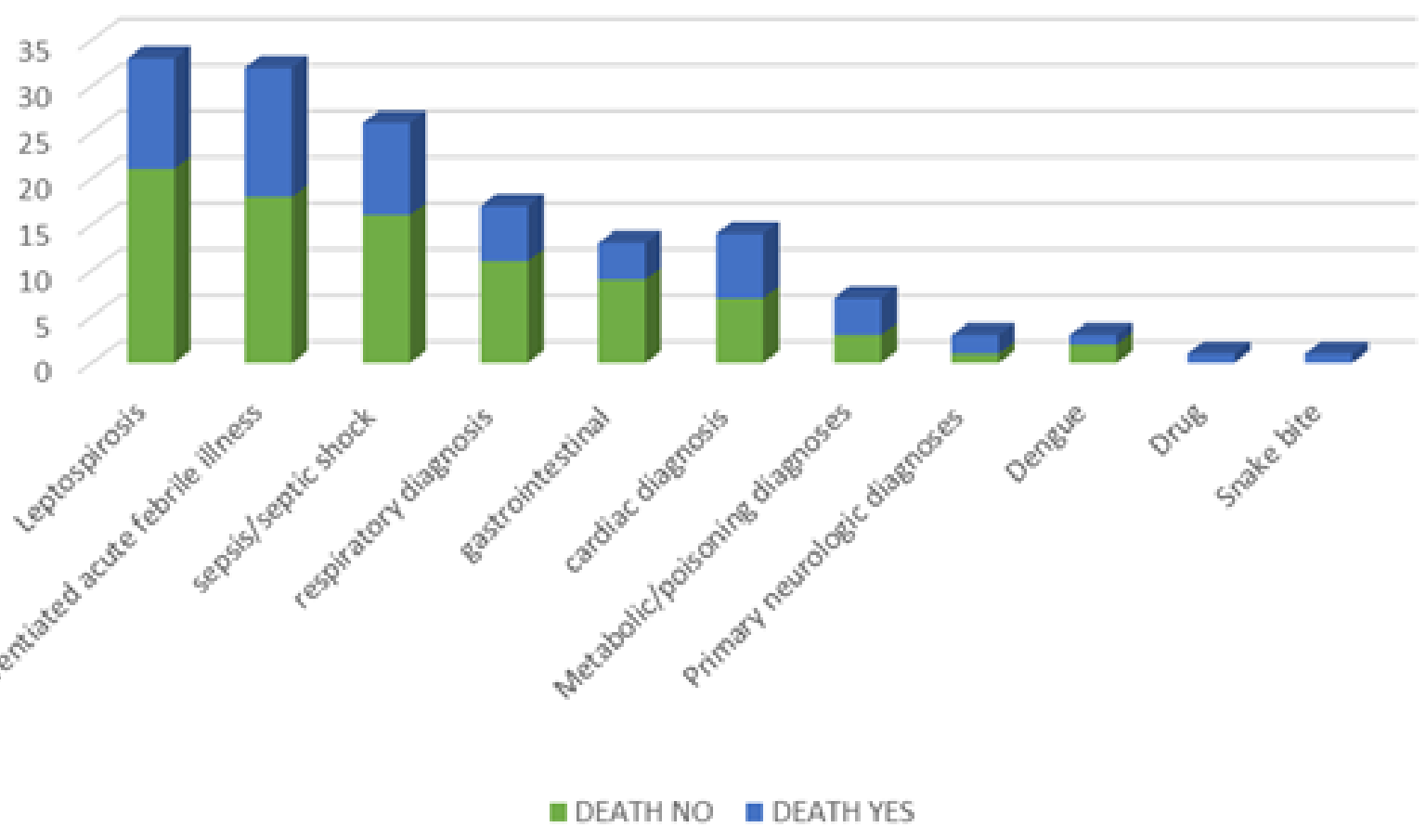

Figure 7. Association between diagnosis and Outcome

Table 10. Association between diagnosis and stage of AKI

\begin{tabular}{|c|c|c|c|c|}
\hline & \multicolumn{3}{|c|}{ AKI stage } \\
\hline & & 1 & 2 & 3 \\
\hline \multirow{22}{*}{ DIAGNOSIS } & \multirow{2}{*}{ Sepsis } & 1 & 8 & 17 \\
\hline & & $3.8 \%$ & $30.8 \%$ & $65.4 \%$ \\
\hline & \multirow{2}{*}{ Cardiac diagnosis } & 2 & 10 & 2 \\
\hline & & $14.3 \%$ & $71.4 \%$ & $14.3 \%$ \\
\hline & \multirow{2}{*}{ Respiratory diagnosis } & 2 & 7 & 8 \\
\hline & & $11.8 \%$ & $41.2 \%$ & $47.1 \%$ \\
\hline & \multirow{2}{*}{ Gastrointestinal } & 0 & 5 & 8 \\
\hline & & & $38.5 \%$ & $61.5 \%$ \\
\hline & \multirow{2}{*}{$\begin{array}{c}\text { Metabolic/poisoning } \\
\text { Diagnoses }\end{array}$} & 0 & 5 & 2 \\
\hline & & & $71.4 \%$ & $28.6 \%$ \\
\hline & \multirow{2}{*}{$\begin{array}{c}\text { Primary neurologic } \\
\text { Diagnoses }\end{array}$} & 1 & 2 & 0 \\
\hline & & $33.3 \%$ & $66.7 \%$ & $0.0 \%$ \\
\hline & \multirow{2}{*}{$\begin{array}{c}\text { Undifferentiated acute } \\
\text { Febrile illness }\end{array}$} & 1 & 3 & 28 \\
\hline & & $3.1 \%$ & $9.4 \%$ & $87.5 \%$ \\
\hline & \multirow{2}{*}{ Leptospirosis } & 0 & 3 & 30 \\
\hline & & & $9.1 \%$ & $90.9 \%$ \\
\hline & \multirow{2}{*}{ Dengue } & 0 & 2 & 1 \\
\hline & & & $66.7 \%$ & $33.3 \%$ \\
\hline & \multirow{2}{*}{ Drug } & 0 & 0 & 1 \\
\hline & & & & $100.0 \%$ \\
\hline & \multirow{2}{*}{ Snake bite } & 0 & 1 & 0 \\
\hline & & & $100.0 \%$ & \\
\hline \multirow{2}{*}{\multicolumn{2}{|c|}{ Total }} & 7 & 46 & 97 \\
\hline & & $4.7 \%$ & $30.7 \%$ & $64.7 \%$ \\
\hline
\end{tabular}


Table 11. Logistic regression analysis of outcome predictors

\begin{tabular}{lllllll}
\hline & & S.E. & Wald & df & Sig. & Exp(B) \\
Basal crepitations & -.416 & .654 & .405 & 1 & .525 & .660 \\
Breathlessness & .989 & .643 & 2.364 & 1 & .124 & 2.687 \\
AKI stage & 20.905 & 5237.667 & .000 & 1 & .997 & 1199225957.801 \\
Ventilation & 3.535 & .726 & 23.717 & 1 & .000 & 34.291 \\
Inotrope & 2.102 & 1.043 & 4.059 & 1 & .044 & 8.186 \\
Indication for dialysis & -.170 & .118 & 2.050 & 1 & .152 & .844 \\
Constant & -66.870 & 15713.000 & .000 & 1 & .997 & .000 \\
\hline
\end{tabular}

\section{MORTALITY}

Mortality was $41 \%$ in the patients in the study group. The ICU mortality rate was 39.3\% (44/112) in study by Medve et al [18] which is comparable with our study.

Maximum mortality was seen in the age group 55-65years $-22.6 \%$ and $45-55$ years. - $17.7 \%$. Mortality was more, $72.6 \%$ in the male patients in this study group compared to females. However, no significant association was identified between age group, gender, patients with comorbidities, and mortality. Age, gender or the presence of co-morbidities was not associated with a higher risk of AKI as in Wijikerma et al [13]

Among those with breathlessness as presenting symptom, $58.8 \%$ expired compared to only $26.8 \%$ among those with no breathlessness on admission to the Medical ICU. Significant association was identified mortality and breathlessness as the presenting symptom. Among those with Basal Crepitations, $53.3 \%$ expired compared to only $33.3 \%$ among those with no Basal Crepitations on admission to the Medical ICU. Significant association was identified.

Respiratory involvement, along with AKI had statistically significant chances of poor outcome $(p<0.05)$ in a study by Bhadade et al [20]

Mortality was more in the patients in this study with undifferentiated acute febrile illness, $43.8 \%$ and leptospirosis, 36.4\%. No significant association was identified between the etiology of AKI and mortality unlike in study by Eswarappa et al which identified significant difference in mortality due to medical versus surgical etiology of AKI.

$53 \%$ of those with stage 3 AKI expired compared to no mortality in the stage 1 or 2 . Significant association could be identified between stage of AKI and mortality. AKIN stages based on creatinine criteria predicted mortality as in a study by Lopez et al. [21]

Mortality was more in the patients in this study who underwent dialysis $48.5 \%$ compared to those who did not require it. This may be because of the fact that they are more hemodynamically compromised. But no significant association was identified between dialysis and mortality.

The need for dialysis treatment has been associated with a higher mortality rate (50-70\%) than among patients with AKI who did not require RRT in study by Bhadade et al [22]

Significant association was identified between indication for dialysis and mortality. $60 \%$ of the patients who underwent dialysis and died had both metabolic acidosis and anuria. Significant association was identified between patients with metabolic acidosis and anuria and mortality in Kumar et al. [23]
Mortality was more in the patients in this study requiring inotrope support (48\%) and ventilator support (70.2\%). Significant association was identified between inotropic and ventilator support; and mortality. Vasopressor support and assisted ventilation were predictors for mortality in a study by Mathew George et al. [24]

By multivariate logistic regression analysis of the factors having association with mortality, requirement of ventilator support and inotropic supports were independent predictors of mortality. Significant association was identified between patients with ventilator support and mortality in Saxena et al ${ }^{47}$ and Liano et al. [25]

\section{CONCLUSION}

150 consecutive patients diagnosed to have acute kidney injury admitted to the medical ICU of a tertiary care center in Kerala were studied. The main burden of diseases were due to infective etiologies. The most common was leptospirosis $(22 \%)$ followed by undifferentiated fever (21\%) and sepsis $(17 \%)$. This is unlike studies done in developed countries. This reflects mainly the trend in tropical regions with higher rainfall. Mortality was $41 \%$ in the study and requirement of ventilator support and inotropic supports were independent predictors of mortality.

In the tropics, diseases like leptospirosis have a high propensity to be the cause of AKI. So a high degree of suspicion should be kept to aid in the diagnosis and early referral to centers with intensive care facilities.

\section{Appendix-1}

\section{LIST OF ABBREVIATION}

1. AKI - Acute Kidney Injury

2. AKIN - Acute Kidney Injury Network

3. CKD - Chronic kidney disease

4. CRRT - Continuous renal replacement therapy

5. ECG - Electro Cardio Gram

6. ELISA - Enzyme Linked Immunosorbant Assay 7. ICU - Intensive care unit

8. INR - International Normalized Ratio

9. KDIGO - Kidney Disease Improving Global Outcome 
10. qSOFA Score - Quick Sequential Organ Failure Assessment

11. RIFLE - Risk of renal failure, Injury to kidney, Failure of kidney function, Loss of kidney function, and Endstage kidney disease

12. RRT - Renal Replacement Therapy

13. S Cr - Serum creatinine

14. SD - Standard deviation

\section{REFERENCES}

[1] Eswarappa M, Gireesh M, Ravi V, Kumar D, Dev G. Spectrum of acute kidney injury in critically ill patients: A single center study from South India. Indian Journal of Nephrology. 2014;24(5):280-280.

[2] Singbartl K, Kellum J. AKI in the ICU: definition, epidemiology, risk stratification, and outcomes. Kidney International. 2012;81(9):819-825.

[3] Bagshaw SM. Septic Acute Kidney Injury in Critically Ill Patients: Clinical Characteristics and Outcomes. Clinical Journal of the American Society of Nephrology. 2007;2:431439.

[4] Prakash J, Murthy AS, Vohra R, Rajak M, Mathur SK. Acute renal failure in intensive care unit (ICU). JAPI. 2006;54:724-728.

[5] Acute Renal Failure in Critically Ill Patients. A Multinational, Multicenter Study. JAMA. 2005;294(7):813-813.

[6] Barrantes F, Tian J, Vazquez R, Amoateng-Adjepong Y, Manthous C. Acute kidney injury criteria predict outcomes of critically ill patients*. Critical Care Medicine. 2008;36(5):1397-1403.

[7] Sushrut S, Waikar JV, Bonventre. Acute kidney injury. Harrison's principles of internal medicine. New York: McGraw Hill; 2011. 18th edition.

[8] Ali T, Khan I, Simpson W, Prescott G, Townend J, Smith W. Incidence and Outcomes in Acute Kidney Injury: A Comprehensive Population-Based Study. Journal of the American Society of Nephrology. 2007;18(4):1292-1298.

[9] Khan J, Khalid S, Khan R, A. Epidemiology of Acute Kidney Injury in the Intensive Care Unit. Critical Care Research and Practice. 2013;2013:1-9.

[10] Cruz D, Bolgan I, Perazella M, Bonello M, Cal MD, Corradi V. North East Italian Prospective Hospital Renal Outcome Survey on Acute Kidney Injury (NEiPHROS-AKI): Targeting the Problem with the RIFLE Criteria. Clinical Journal of the American Society of Nephrology. 2007;2(3):418-425.

[11] Bandara K, Weerasekera M, Gunasekara C, Ranasinghe N, Marasinghe C, Fernando N. Utility of modified Faine's criteria in diagnosis of leptospirosis. BMC Infectious Diseases. 2016;16(1).

[12] Wijewickrama E, Ratnayake G, Wikramaratne C, Sheriff $\mathrm{R}$, Rajapakse S. Incidences and clinical outcomes of acute kidney injury in ICU: a prospective observational study in Sri Lanka. BMC Research Notes. 2014;7(1):305-305.

[13] Basu G, Chrispal A, Boorugu H, Gopinath KG, Chandy S, Prakash JA. Acute kidney injury in tropical acute febrile illness in a tertiary care centre-RIFLE criteria validation. Nephrol Dial Transplant. 2011;26:524-555.
[14] F, PJ. The Madrid Acute Renal Failure Study Group. Epidemiology of acute renal failure: A prospective, multicenter, community-based study. Kidney International. 1996;50(3):811-818.

[15] Ede FD, Silva J, Vieira GB, Souza AP, JB, Fdos F, et al. Acute kidney injury in a tropical country: A cohort study of 253 patients in an infectious diseases Intensive Care Unit. Rev Soc Bras Med Trop. 2014;47:86-95.

[16] Chugh KS, Sitprija V, Jha V, Davidson AM, Cameron JS, Grunfeld JP, et al. Acute renal failure in tropical countries. Oxford textbook of clinical nephrology. 2005;p. 639-58. 3rd edition.

[17] Medve L, Antek C, Paloczi B, Kocsi S, Gartner B, Marjanek Z. Epidemiology of acute kidney injury in Hungarian intensive care units: a multicenter, prospective, observational study. BMC Nephrol. 2011;13:12-43.

[18] Angus DC, Linde-Zwirble WT, Lidicker J, Clermont G, Carcillo J, Pinsky MR. Epidemiology of severe sepsis in the United States: analysis of incidence, outcome, and associated costs of care. Crit Care Med. 2001;29:1303-1313.

[19] Jha V, Chugh KS. Community-acquired acute kidney injury in Asia. Semin Nephrol. 2008;28(4):330-377.

[20] Mathew AJ, George J. Acute kidney injury in the tropics. Ann Saudi Med. 2011;31(5):451-457.

[21] Kumar S, Raina S, Vikrant S, Patial RK. Spectrum of acute kidney injury in the Himalayan region. Indian journal of Nephrology. 2012;22(5):363-366.

[22] Ibrahim A, Ahmed M, Kedir S, Bekele D. Clinical profile and outcome of patients with acute kidney injury requiring dialysis-an experience from a haemodialysis unit in a developing country. BMC Nephrology. 2016;17(1).

[23] Bhadade R, De, Souza R, Harde M, Mehta K, Bhargava P. A Prospective Study of Acute Kidney Injury According to KDIGO Definition and its Mortality Predictors. Journal of The Association of Physicians of India. 2016;64:22-28.

[24] Lopes J, Fernandes P, Gonçalves JS, Alvarez S, A, Silva C, et al. Acute kidney injury in intensive care unit patients: a comparison between the RIFLE and the Acute Kidney Injury Network classifications. Critical Care. 2008;12(4):110110.

[25] Daher EDF, Soares D, Fernandes ADM, Girão M, Sidrim $\mathrm{P}$, Pereira E. Risk factors for intensive care unit admission in patients with severe leptospirosis: a comparative study according to patients' severity. BMC Infectious Diseases. $2015 ; 16(1)$. 